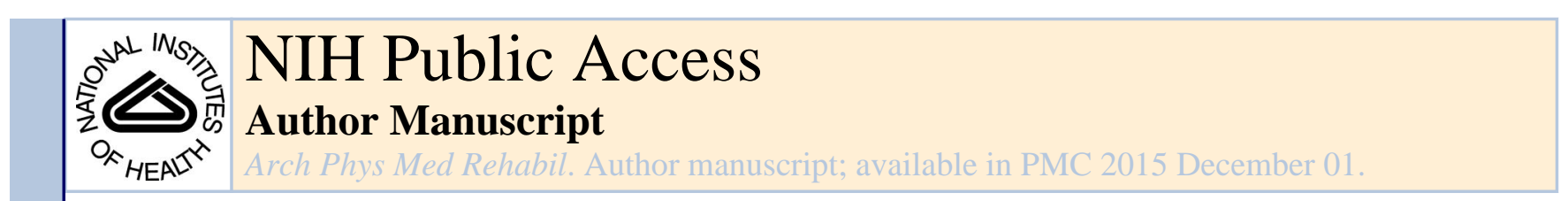

Published in final edited form as:

Arch Phys Med Rehabil. 2014 December ; 95(12): 2484-2490. doi:10.1016/j.apmr.2014.04.028.

\title{
Task Difficulty and Life Changes among Stroke Family Caregivers: Relationship to Depressive Symptoms
}

\author{
Susan M. McLennon, PhD, RN ${ }^{1}$, Tamilyn Bakas, PhD, RN, FAHA, FAAN ${ }^{1}$, Nenette M. \\ Jessup, MPH, CCRP ${ }^{1}$, Barbara Habermann, PhD, RN, FAAN ${ }^{2}$, and Michael T. Weaver, PhD, \\ RN, FAAN ${ }^{1}$ \\ ${ }^{1}$ Indiana University, School of Nursing \\ ${ }^{2}$ University of Delaware
}

\begin{abstract}
OBJECTIVE-To investigate differences in stroke caregiver task difficulty and life changes based on level of caregiver depressive symptoms, and to estimate probabilities among task difficulty and life change items.
\end{abstract}

DESIGN—Descriptive analysis of baseline data from an ongoing stroke caregiver intervention trial.

SETTING-Caregivers recruited from 10 mid-western hospitals and rehabilitation facilities.

PARTICIPANTS-Caregivers ( $\mathrm{N}=242 ; 78.6 \%$ female; $47.7 \%$ spouses; $71.8 \%$ white; mean age $54.2 \pm 12.1$ ) caring for stroke survivors within 8 weeks of discharge to home.

INTERVENTIONS-Not applicable.

MAIN OUTCOME MEASURES-Baseline measures for task difficulty (Oberst Caregiving Burden Scale) and life changes (Bakas Caregiving Outcomes Scale) were compared based on level of depressive symptoms (PHQ-9 scores $<5=$ no depressive symptoms, $n=126$; PHQ-9 scores $\geq 5$ $=$ mild to severe depressive symptoms, $\mathrm{n}=116$ ). Mean scores were analyzed using general linear modeling, with item analyses using logistic regression and the Benjamini-Hochberg method to control Type I error inflation.

RESULTS-Caregivers with mild to severe depressive symptoms had greater difficulty with tasks and worse life changes than those with no depressive symptoms ( $\mathrm{p}<.001)$. Odds ratios were highest for the task of arranging care while away, and highest for negative life changes such as addressing self-esteem, coping with stress, and physical health.

() 2014 The American Congress of Rehabilitation Medicine. Published by Elsevier Inc. All rights reserved.

Primary author contact information: Susan M. McLennon, PhD, RN Assistant Professor Indiana University, School of Nursing 1111 Middle Dr. NU W437 Indianapolis, IN 46202 smclenno@iupui.edu 317-278-0459 (o) 317-564-4494 (h).

Publisher's Disclaimer: This is a PDF file of an unedited manuscript that has been accepted for publication. As a service to our customers we are providing this early version of the manuscript. The manuscript will undergo copyediting, typesetting, and review of the resulting proof before it is published in its final citable form. Please note that during the production process errors may be discovered which could affect the content, and all legal disclaimers that apply to the journal pertain.

Findings presented (podium) at the American Heart Association/International Stroke Conference, February 2013, Honolulu, HA.

The authors have no conflicts of interest. 
CONCLUSION-Findings underscore the importance of depressive symptom screening for stroke caregivers during or shortly after discharge. Assisting caregivers with depressive symptoms to arrange for respite care and addressing negative physical and psychological changes may be priority areas for future interventions.

\section{Keywords}

Family Caregiving; Oberst Caregiving Outcomes Scale; Bakas Caregiving Outcomes Scale; Depressive Disorder; PHQ-9

In the last decade, hospital admissions for patients with strokes have increased by $18 \%{ }^{1}$ Costs associated with the 1,015,000 stroke survivors discharged from hospitals in 2010 exceeded $\$ 22$ billion. ${ }^{2}$ Most stroke survivors (68\%-74\%) were discharged to home with a family member providing care, although family caregivers are often unprepared for the challenges of caring for a person with impairments at multiple levels. ${ }^{3-6}$ Common impairments after a stroke include alterations in mobility, behavior, mood, and cognition, as well as speech and sensory loss. Mobility deficits typically involve residual limb paralysis or weakness (50\%), difficulty ambulating independently (30\%), and the need for assistance with routine daily activities (25\%). ${ }^{7}$ Behavior and mood problems of the stroke survivor are associated with caregiver perceptions of task difficulty, life changes, depressive symptoms, and caregiver stress. ${ }^{8,9}$ The demands of caregiving increase the risk of caregivers neglecting their own health and experiencing psychological sequelae such as depressive symptoms, increasing the risk for institutionalization of the stroke survivor. ${ }^{10,11}$

As many as $75 \%$ of stroke caregivers report unmet needs and concerns the first few months at home because of lack of training and ability to provide care. ${ }^{12,13}$ In interviews with 14 family stroke caregivers, researchers identified role strain as a primary barrier. ${ }^{14}$ Empirical findings from a literature review of 23 articles suggested that stroke family caregivers' needs are not adequately assessed or addressed prior to discharge, a strategy that if implemented routinely could promote more positive outcomes for survivors and their families. ${ }^{15}$

Many factors may potentially influence stroke caregivers' abilities to manage caregiving demands after the survivor's discharge from the hospital or rehabilitation. In most situations, it is important that caregivers have sufficient physical and psychological health to cope with these demands and enable the stroke survivor to remain at home. In particular, the presence of depressive symptoms is an important factor to assess when considering caregivers' abilities to provide care at home for the stroke survivor because of the potential negative impact on the caregiver as well as the survivor. ${ }^{16,17}$

As many as $52 \%$ of stroke family caregivers have been found to exhibit depressive symptoms with some studies reporting more depressive symptoms in caregivers than in survivors. ${ }^{18-20}$ Stroke caregiver depressive symptom risk is greater earlier in the caregiving trajectory, in caregivers who report greater burden and more negative life changes as a result of becoming a caregiver, and in female caregivers ${ }^{10,21-23}$ It is also possible that caregivers may have clinical depression or depressive symptoms prior to becoming a caregiver. Thus, assessing family caregivers for depressive symptoms early in the caregiving trajectory may be important. 
Recent recommendations from the American Heart Association included the need to screen for depressive symptoms in stroke survivors using the Patient Health Questionnaire (PHQ-9) depression. ${ }^{24-26}$ However, screening caregivers with the PHQ-9 might also assist health care providers to identify those at risk for greater difficulties with caregiving who may need closer monitoring during the early phase of caregiving. ${ }^{6,26}$ The purposes of this study were to a) determine differences in mean task difficulty and life changes total scores based on caregiver depressive symptoms as measured by the PHQ-9; and b) estimate the probability of experiencing specific caregiving difficulty and negative life changes during the early discharge period.

\section{Methods}

\section{Sample}

Baseline data from participants in the parent Telephone Assessment and Skill-Building Intervention Study (TASK II) (R01 NR010388), an ongoing stroke caregiver intervention trial, were used for this descriptive analysis. Developed from findings from an earlier study detailed elsewhere, the purpose of the TASK II trial is to test the efficacy of the TASK II Intervention group compared to an Information, Support, and Referral group in family caregivers of stroke survivors. ${ }^{27}$ Participants were stroke caregivers providing care for survivors residing in the community who were recruited from one of 10 participating midwestern hospitals or rehabilitation facilities. Caregivers were considered eligible if they were over age 21, were the primary caregiver (unpaid family member or significant other) of the stroke survivor, had been providing care for eight weeks or less after the survivor was discharged home, planned to be providing care for at least one year, had access to a telephone, and were willing to participate in study calls at designated time points. For the present study, data collected during baseline telephone interviews with stroke family caregivers prior to randomization were analyzed.

\section{Procedures}

Institutional Review Board approval was received and the study procedures followed standard ethical guidelines for the protection of human subjects in research including informed consent and data safety and monitoring. Potential participants were mailed study information packets including informed consent materials prior to the scheduled baseline data collection interview which was conducted within eight weeks of discharge to the home setting. Data were collected by trained research assistants who followed a standard script when contacting the stroke caregivers by telephone. All data collectors were routinely monitored for adherence to data collection protocols.

\section{Measures}

The Oberst Caregiving Burden Scale (OCBS) difficulty subscale was used to determine perceived task difficulty. ${ }^{28}$ The Bakas Caregiving Outcomes Scale (BCOS) was used to measure perceived life changes. ${ }^{29}$ The Patient Health Questionnaire (PHQ-9) was used to assess level of depressive symptoms, and the Stroke Survivor Quality of Life Scale (SSQOL) was used to evaluate stroke survivor functioning and cognition. ${ }^{24,30}$ Data were 
collected from a demographic survey and the Chronic Conditions Index (CCI) to describe the sample and assess general health status. ${ }^{31}$

Perceived task difficulty includes tasks such as providing personal care, assisting with mobility, watching for symptoms, managing emotions and behaviors, providing transportation, dealing with finances, and talking with health care providers, among others. ${ }^{28}$ The OCBS measures caregiver perceptions about the dimensions of time and difficulty for 15 different tasks common to stroke caregivers. For this study, the summed ratings for each of the 15 items related to the dimension of task difficulty were used (not difficult $=1$ to extremely difficult $=5$ ). Higher total scores represented greater perceived difficulty with tasks. Evidence for content and construct validity has been reported in samples of cancer caregivers. ${ }^{32-33}$ Acceptable internal consistency reliability $(\alpha=.84-.97)$ in the context of family caregivers for cancer patients and stroke survivors has also been reported. ${ }^{12,29,34}$ The Cronbach's alpha for the OCBS task difficulty subscale in the current sample was 0.90 . Tasks such as providing personal and medical care, assisting with activities of daily living, monitoring symptoms, managing the patient's emotions and behaviors, dealing with finances, and coordinating and seeking health services are included on the OCBS scale.

Life changes refer to changes in social functioning, subjective well-being, and physical health specifically as a result of providing care. ${ }^{29}$ The Bakas Caregiving Outcomes Scale (BCOS), revised was used to assess life changes as a result of caregiving. ${ }^{29}$ It consists of 15 items, each item with a possible score range from 1 (changed for the worst) to 7 (changed for the best). Sample items include changes in time for family and social activities, emotional well-being, ability to cope with stress, level of energy, physical functioning, and general health, among others. The range for total scores is 15 to 105 , with scores averaging over 60 being "changed for the best" and those lower than 60 as "changed for the worst." Lower scores indicate more negative perceived care-related life changes. Construct and criterion-related validity, as well as internal consistency reliability $(a=.90)$ in stroke caregivers have been previously reported. ${ }^{29}$ The Cronbach's alpha for the BCOS for the current sample was 0.87 . The BCOS addresses changes in social functioning, subjective well-being, and health, specifically as a result of providing care. ${ }^{29}$

Depressive symptoms are those defined by the nine DSM-IV criteria for depression (i.e., little interest or pleasure; feeling down or depressed; trouble sleeping; feeling tired or having little energy; poor appetite or overeating; feeling bad about oneself; trouble concentrating; moving or speaking slowly; thoughts about being better off dead or hurting self in some way). ${ }^{24}$ The Patient Health Questionnaire (PHQ-9) depressive symptom scale, used to assess caregiver depressive symptom severity, is a 9-item measure with application in clinical and research settings. ${ }^{24}$ In clinical settings it can be used by qualified clinicians as an indicator of depression. The tool incorporates symptom frequency into the scoring index and also screens for suicidal ideation. Items address each of the nine DSM-IV criteria for depression, and participants score each item based on how much they have been bothered by the problem, from 0 (not at all) to 4 (nearly every day). Total scores can range between 0 (no depressive symptoms) and 27 (severe depressive symptoms), with suggested ranges for depressive symptom severity as follows: no (0-4), mild (5-9), moderate (10-14), moderately 
severe (15-19), and severe (20-27). For this study, PHQ-9 scores were dichotomized into two groups: no depressive symptoms (PHQ-9 < 5) and mild to severe depressive symptoms (PHQ-9 $\geq 5$ ). We did not have sufficient subgroup sizes to analyze all five levels of depressive symptoms separately (no, mild, moderate, moderately-severe, and severe). Evidence for scale reliability in primary care and stroke caregivers has been reported $(\alpha=$. 80 to .89$).{ }^{6,24,29}$ The Cronbach's alpha for the PHQ-9 for the current sample was 0.82 .

A demographic survey assessed characteristics of the stroke caregiver and survivor such as age, gender, race, and type of relationship. Two domains from the Stroke Survivor Quality of Life Scale (SS-QOL) were used to describe mobility and cognition of the stroke survivors, as perceived by the caregivers. ${ }^{30}$ The SS-QOL is a 49-item measure with 12 domains to assess health-related quality of life of patients with stroke and may be completed by proxy. ${ }^{30,35}$ Items are rated on a 5-point Likert scale with higher scores indicating better functioning. The Cronbach's alpha for the SSQOL mobility and cognition subscales for the current sample was 0.94 . The Chronic Conditions Index contains 13 items to quantify the number of chronic health conditions as an indicator of health status. ${ }^{31}$ Stroke caregivers in this study were asked about their own and the survivor's chronic conditions using responses of "yes" or "no" to indicate the presence or absence of the condition.

\section{Statistical Analyses}

SAS version 9.3 was used for the statistical analysis. ${ }^{34}$ Descriptive statistics (frequencies, percentages, means, and standard deviations) appropriate for scales were used to characterize the sample. A total of 242 caregivers had complete PHQ-9 scores, and were dichotomized into two groups: no depressive symptoms (PHQ-9 < 5) and mild to severe depressive symptoms (PHQ-9 25 ). Differences in means between the depressive symptom groups on selected potential covariates, namely caregiver and stroke survivor ages, gender, caregiver and stroke survivor number of chronic conditions, and survivor mobility and cognition were tested using t-tests. Contingency tables and chi squared with exact $p$ values were used to test for differences in distribution of caregiver gender, race, and relation. ${ }^{36}$ Variables that demonstrated differences between the depressive symptoms groups were utilized as covariates. Separate general linear models (GLM) were used to test for differences in mean Task Difficulty (OCBS) and Life Changes (BCOS) between the depressive symptom groups, after controlling for the selected covariates. To examine differences in distribution for individual OCBS and BCOS items between the depressive symptom groups, responses for individual OCBS and BCOS items were dichotomized as follows: OCBS items were dichotomized into Not/Slightly Difficult (1-2) or Moderate/Very/ Extremely Difficult (3-5); BCOS items were dichotomized into Life Change for the Worse (1-3) or No/Beneficial Change (4-7). Individual logistic regression models were used to test for differences in probability of endorsing an adverse (moderate/very/extremely difficult task or life change for the worse) response between depressive symptoms groups, after controlling for the selected covariates. Conformance to statistical assumptions was examined for each model, and appropriate remedial measures applied where required. The HosmerLemeshow goodness of fit test was examined for each logistic regression model. ${ }^{37}$ The Benjamini-Hochberg method was used to maintain a $5 \%$ false discovery rate within each set of OCBS and BCOS items. ${ }^{38,39}$ 


\section{Results}

Descriptive statistics for the sample are presented in Table 1. Most of the 242 stroke caregivers were white, female, with a mean age of 54.2 years. There were slightly more nonspouse caregivers (52\%) than spouse caregivers (48\%). The stroke survivors were slightly older with a mean age of 63.0 years, and moderately mobility and cognitively impaired. Family caregivers reported an average of 2.2 chronic health conditions compared to 4.08 for the stroke survivors. There were 126 caregivers with PHQ-9 scores less than 5, who were classified as having no depressive symptoms and 116 caregivers with PHQ-9 scores equal to or greater than 5 who were classified as having mild to severe depressive symptoms.

Nine variables were examined for use as covariates in the general linear model (GLM) and logistic models. Results of the bivariate tests are presented in Table 2. Four variables, namely caregiver chronic conditions and gender, and survivor mobility and cognition, exhibited differences between the two depressive symptom groups and therefore were included in the GLM and logistic regression models.

Results of the GLM analyses testing differences in mean perceived caregiving difficulty and life changes appear in Table 3 . In terms of task difficulty, the overall model was statistically significant ( $\mathrm{F}=14.5 ; \mathrm{DF}=5,235 ; \mathrm{p}<.001)$. Participants with mild to severe depressive symptoms had higher $(\mathrm{F}=16.6 ; \mathrm{DF}=1,235 ; \mathrm{p}<.001)$ least square mean values (33.6) than those with no depressive symptoms (27.8), after controlling for covariates in the model. The overall model for life changes was also statistically significant $(F=12.4 ; D F=5,234 ; p<.001)$. Participants with mild to severe depressive symptoms had lower $(\mathrm{F}=25.3 ; \mathrm{DF}=1,234 ; \mathrm{p}<$. 001) least square mean values (52.4) than participants with no depressive symptoms (59.0), after controlling for covariates in the model.

Selected logistic regression model results for individual task difficulty (OCBS) and life change (BCOS) items are presented in Table 4. Applying the Benjamini-Hochberg false discovery rate adjustment method, the mild to severe depressive symptom group participants had higher odds for endorsing one OCBS and 10 BCOS items, after controlling for covariates in the model. Specifically, the mild to severe depressive symptom group participants were found to be more likely $(\mathrm{OR}=3.46, \mathrm{p}<.001)$ to endorse having difficulty with "finding and arranging for someone to care for the patient while away" (OCBS item 12), after controlling for caregiver chronic conditions and gender, and survivor mobility and cognition. In terms of the BCOS, the 10 items encompassed a range of psychological, social, and physical factors: self-esteem, ability to cope with stress, physical health, emotional wellbeing, financial well-being, level of energy, general health, roles in life, future outlook, and physical functioning.

\section{Discussion}

In this study, baseline data from family stroke caregivers participating in a stroke intervention trial, the Telephone Assessment and Skill-Building Intervention Study (TASK II) (R01 NR010388), were used to determine differences in mean caregiver task difficulty and life changes and to estimate the probability of experiencing specific caregiving task 
difficulties and negative life changes during the first eight weeks after the stroke survivor was discharged. Of the 242 caregivers, $47 \%$ reported mild to severe depressive symptoms, similar to that reported by others. ${ }^{18,20}$ The average depressive symptom scores in this sample were in the mild range during the first eight weeks after discharge. Overall, the caregivers considered caregiving tasks to be moderately difficult and they perceived moderately great negative life changes as a result of becoming a stroke caregiver.

Caregiver chronic conditions and gender, and caregiver perceptions of stroke survivor mobility and cognition were associated with caregiver depressive symptoms. Family stroke caregivers in this study reported an average of 2.2 chronic health conditions. Those conditions may have reduced caregiver physical and/or emotional capacity through added demands on top of caring for the stroke survivor. Since stroke survivors required assistance with an average of 4.08 health conditions, the caregivers were potentially directly or indirectly experiencing a total of six chronic conditions. As the population ages, the number of older caregivers who have multiple health conditions and associated physical limitations is expected to increase. ${ }^{40}$ Conditions such as arthritis, hypertension, fatigue, headaches, insomnia, and joint pain may negatively impact the ability of family caregivers to provide care. $^{40-42}$

There were gender differences between the two depressive symptom groups; female caregivers had more depressive symptoms. In the stroke literature, the relationship of gender to caregiving outcomes is mixed. Similar to our findings, some have suggested that female caregivers are at greater risk for depressive symptoms. ${ }^{6,23}$ In another study, male stroke caregivers experienced greater negative effects when caring for their wives, whereas others have reported no gender differences in caregiving outcomes. ${ }^{43-44}$ Differences in research findings related to stroke caregiver gender have likely been complicated by variations in sampling (e.g., size, types of relationships between the caregiver and survivor), lack of measurement consistency across studies, and issues of change over time (e.g., crosssectional vs. longitudinal studies).

Caregiver perceptions of impairments in survivor mobility and cognition were associated with more caregiver depressive symptoms. In this sample, caregivers perceived stroke survivors to be moderately impaired in both mobility and cognition. Similarly, others have reported that impairments in the stroke survivor contribute to caregiver strain and burden. ${ }^{43,45}$ Longitudinal research designs that follow caregivers over the caregiving trajectory may help to elucidate the relationship between caregiver depressive symptoms and proxy ratings of stroke survivor functioning.

These findings offer evidence for the need to provide emotional support for caregivers of a stroke survivor with impaired memory and lower functional ability. ${ }^{46}$ When considering these factors in the context of the aging population, the importance of preserving the physical and psychological health of caregivers cannot be overestimated. A major strength of this study was that by controlling for the potential influence of the four covariates, the central role of depressive symptoms among stroke caregivers was illuminated. 
Main study findings indicated that stroke caregivers in the mild to severe depressive symptom group perceived greater caregiving task difficulties (OCBS) and more negative life changes (BCOS) than those without depressive symptoms, controlling for caregiver chronic conditions and gender, and stroke survivor mobility and cognition. The final model testing for perceived task difficulty accounted for $23.6 \%$ of the variance, suggesting additional factors not included in this study also influenced the overall caregiving context. Although only one OCBS item, "arranging care while away", met the Benjamini-Hochberg criteria, seven others exhibited $p$ values of less than .05. Though this test offers the advantage of good control of Type I error, a disadvantage is a potentially higher rate of Type II errors, that is, of assuming no relationship between variables when in fact there is one.

Consequently, the other 7 items should not be summarily discarded, but rather considered in context. Ongoing care of stroke survivors when their family caregivers have depressive symptoms should include assessing for potential difficulties in these key areas, with greater attention given to assisting and encouraging caregivers to arrange for routine respite care. Caregivers without adequate resources (e.g., family, friends, financial) for respite care would benefit from efforts directed toward policy change at the federal or state levels to subsidize respite care for caregivers. Future studies may provide additional information about the specific caregiving tasks that are difficult for stroke caregivers in the early postdischarge period.

There were differences in caregivers' perceived life changes between the two depressive symptom groups. Caregivers with mild to severe depressive symptoms reported more negative life changes because of providing care for the stroke survivor, when caregiver gender and health and survivor mobility and cognition were controlled. The final model accounted for $21 \%$ of the variance, suggesting that additional factors contributed to the overall caregiving context. Though 10 of the BCOS items met the Benjamini-Hochberg criteria, four others exhibited $\mathrm{p}$ values of less than .05. The implications of good control for Type I error at the risk of greater Type II error are similar here, and therefore those four items should also be considered in context. For those with depressive symptoms, it is noteworthy that so many areas in their lives were seen as negatively affected. This suggests that it may be more than the difficulty of the tasks that is problematic, rather it may be the overall toll of caregiving on their lives that is especially harmful.

Whether caregivers had pre-existing depressive symptoms, or the depressive symptoms arose as a result of becoming a caregiver, or both, these results highlight the importance of early screening for caregiver depressive symptoms. It is not surprising that caregivers with depressive symptoms also had a greater risk for experiencing lower self-esteem, less ability to cope with stress, and poorer physical health and emotional well-being. Targeting interventions to assist caregivers in the areas identified as particularly problematic may be especially helpful for caregivers and ultimately offer benefit to the stroke survivors as well.

\section{Study Limitations}

There are several limitations to consider when interpreting the results of this study. First, it is possible that some caregivers may have exhibited clinical depression or depressive symptoms prior to becoming a caregiver. Therefore, it cannot be assumed that caregiver task 
difficulty and life changes resulted only from newly emerging caregiver depressive symptoms as a result of providing care. However, caregiver depressive symptoms, whether they existed prior to becoming a caregiver or after, could place caregivers at risk for task difficulty and negative life changes. Identifying caregivers with depressive symptoms during the early discharge period may unveil caregivers with pre-existing clinical depression that requires further treatment, or may identify caregivers with emerging depressive symptoms as a result of providing care.

We also dichotomized depressive symptoms using PHQ-9 scores into two groups: no depressive symptoms (PHQ-9 < 5) and mild to severe depressive symptoms (PHQ-9 25). We did not have sufficient subsample sizes to analyze depressive symptoms across all five subtypes (no, mild, moderate, moderately severe, and severe). Future studies with larger subsample sizes might reveal more clinically helpful information useful to targeting limited resources and provider time.

As the sample was not randomly selected from stroke caregivers, generalizability is limited. Certain characteristics of this sample may have been different from the general population of stroke caregivers. Our sample of stroke caregivers was recruited primarily from large urban hospitals and rehabilitation facilities in the Midwest. They were within two months of discharge of the stroke survivor and thus may not have represented caregivers who had been caring for a stroke survivor over a longer time period. Furthermore, the stroke caregivers in this study who agreed to participate may have been more inclined to seek help than those who did not consent. Finally, no causal conclusions may be drawn from these baseline data taken from a single time point.

\section{Conclusions}

The results of this study suggest that stroke caregivers with depressive symptoms may experience greater task difficulty and more negative life changes during the initial period when assuming care for a stroke survivor at home. Future studies to elucidate causal relationships between depressive symptoms and perceptions of task difficulty and life changes are needed. Stroke caregivers may benefit from screening for depressive symptoms early in the caregiving trajectory. The PHQ-9 has been identified as a clinically useful and efficient method to screen for depressive symptoms. ${ }^{24}$ Of particular importance relative to task difficulty is the need to address caregiver access to respite care. Regarding negative life changes, monitoring for alterations in caregiver self-esteem, ability to cope with stress, emotional well-being, and physical health are recommended.

\section{Acknowledgments}

Funded by the National Institute of Health/National Institute of Nursing Research: Telephone Assessment and Skill-Building Intervention Study (TASK II) (R01 NR010388)

\section{References}

1. Qureshi AI, Mendelow AD, Hanley DF. Intracerebral haemorrhage. Lancet. 2009; 2009; 373:16321644. [PubMed: 19427958] 
2. Go AS, Mozaffarian D, Roger VL, et al. Heart disease and stroke statistics - 2013 update: a report from the American Heart Association. Circulation. 2013; 127:e6-e245. doi: 10.1161/CIR. 0b013e31828124ad. [PubMed: 23239837]

3. Dewey HM, Thrift AG, Mihalopoulos C, et al. Informal care for stroke survivors: Results from the North East Melbourne Stroke Incidence Study (NEMESIS). Stroke. 2002; 33:1028-1033. [PubMed: 11935056]

4. Dorsey MK, Vaca KJ. The stroke patient and assessment of caregiver needs. J Vasc Nurs. 1998; 16:62-67. [PubMed: 9883149]

5. King RB, Ainsworth CR, Ronen M, Hartke RJ. Stroke caregivers: Pressing problems reported during the first months of caregiving. J Neurosci Nurs. 2010; 42(6):302-11. [PubMed: 21207768]

6. Bakas T, Kroenke K, Plue LD, Perkins SM, Williams LS. Outcomes among family caregivers of aphasic versus non-aphasic stroke survivors. Rehabil Nurs. 2006; 31:33-42. [PubMed: 16422043]

7. Kelly-Hayes M, Beiser A, Kase CS, Scaramucci A, D'Agostino RB, Wolf PA. The influence of gender and age on disability following ischemic stroke: the Framingham study. J Stroke Cerebrovasc Dis. 2003; 12(3):119-26. [PubMed: 17903915]

8. Gonzalez C, Bakas T. Factors associated with stroke survivor behaviors as identified by family caregivers. Rehabil Nurs. 2013; 38(4):202-11. [PubMed: 23686515]

9. Haley WE, Allen JY, Grant JS, Clay OJ, Perkins M, Roth DL. Problems and benefits reported by stroke family caregivers: Results from a prospective epidemiological study. Stroke. 2009; 40(6): 2129-33. [PubMed: 19407230]

10. Denno MS, Gillard PJ, Graham GD, et al. Anxiety and depression associated with caregiver burden in caregivers of stroke survivors with spasticity. Arch Phys Med Rehabil. 2013; 94(9):1731-6. [PubMed: 23548544]

11. Han B, Haley WE. Family caregiving for patients with stroke: Review and analysis. Stroke. 1999; 30:1478-1485. [PubMed: 10390326]

12. Bakas T, Burgener SC. Predictors of emotional distress, general health, and caregiving outcomes in family caregivers of stroke survivors. Top Stroke Rehabil. 2002; 9(1):34-35. [PubMed: 14523721]

13. King RB, Semik PE. Stroke caregiving: Difficult times, resource use, and needs during the first 2 years. J Gerontol Nurs. 2006; 32(4):37-44. [PubMed: 16615711]

14. White CL, Mayo N, Hanley JA, Wood-Dauphinee S. Evolution of the caregiving experience in the initial 2 years following stroke. Res Nurs Health. 2003; 26(3):177-89. [PubMed: 12754726]

15. Loupis YM, Faux SG. Family conferences in stroke rehabilitation: A literature review. J Stroke Cerebrovasc Dis. 2013; 22(6):883-93. [PubMed: 23352687]

16. Grant JS, Clay OJ, Keltner NL, et al. Does caregiver well-being predict stroke survivor depressive symptoms? Top Stroke Rehab. 2013; 20(1):44-51.

17. Klinedinst NJ, Gebhardt MC, Aycock DM, et al. Caregiver characteristics predict stroke survivor quality of life at 4 months and 1 year. Res Nurs Health. 2009; 32(6):592-605. [PubMed: 19722169]

18. Berg A, Palomaki H, Lonnqvist J, Lehtihalmes M, Kaste M. Depression among caregivers of stroke survivors. Stroke. 2005; 36:639-643. [PubMed: 15677575]

19. Cameron JL, Cheung AM, Streiner DL, Coyte PC, Stewart DE. Stroke survivor depressive symptoms are associated with family caregiver depression during the first 2 years poststroke. Stroke. 2011; 42(2):302-6. [PubMed: 21164124]

20. Morimoto T, Schreiner AS, Asano H. Perceptions of burden among family caregivers of poststroke elderly in Japan. Int J Rehabil Res. 2001; 24(3):221-6. [PubMed: 11560237]

21. Godwin KM, Ostwald SK, Cron SG, Wasserman J. Long-term health-related quality of life of stroke survivors and their spousal caregivers. J Neurosci Nurs. 2013; 45(3):147-54. [PubMed: 23558977]

22. Peyrovi H, Mohammad-Saeid D, Farahani-Nia M, Hoseini FJ. The relationship between perceived life changes and depression in caregivers of stroke patients. J Neurosci Nurs. 2012; 44(6):329-36. [PubMed: 23124123]

23. Alexander T, Wilz G. Family caregivers: Gender differences in adjustment to stroke survivors' mental changes. Rehabil Psychol. 2010; 55(2):159-69. [PubMed: 20496970] 
24. Kroenke K, Spitzer RL, Williams JBW. The PHQ-9: Validity of a brief depression severity measures. J Gen Intern Med. 2001; 16:606-613. [PubMed: 11556941]

25. Williams LS, Brizendine EJ, Plue L, et al. Performance of the PHQ-9 as a screening tool for poststroke depression. Stroke. 2005; 36:635-638. [PubMed: 15677576]

26. Miller ET, Murray L, Richards L, et al. on behalf of the American Heart Association Council on Cardiovascular Nursing and the Stroke Council. Comprehensive overview of nursing and interdisciplinary rehabilitation care of the stroke patient. A scientific statement from the American Heart Association. Stroke. 2010; 41:2402-2448. [PubMed: 20813995]

27. Bakas T, Farran CJ, Austin JK, Given BA, Johnson EA, Williams LS. Stroke caregiver outcomes from the Telephone Assessment and Skill-Building Kit (TASK). Top Stroke Rehabil. 2009; 16:105-121. [PubMed: 19581197]

28. Bakas T, Austin JK, Jessup SL, Williams LS, Oberst MT. Time and difficulty of tasks provided by family caregivers of stroke survivors. J Neurosci Nurs. 2004; 36(2):95-106. [PubMed: 15115364]

29. Bakas T, Champion V, Perkins SM, Farran CJ, Williams LS. Psychometric testing of the revised 15-item Bakas Caregiving Outcomes Scale. Nurs Res. 2006; 55:346-355. [PubMed: 16980835]

30. Williams LS, Weinberger M, Harris LE, Clark DO, Biller J. Development of a stroke-specific quality of life scale. Stroke. 1999; 30(7):1362-9. [PubMed: 10390308]

31. Cornoni-Huntley, J.; Brock, DB.; Ostfeld, AM. Established populations for epidemiologic studies of the elderly resource data book. U.S. Department of Health and Human Services; Washington: 1986. No. NIH Pub No. 86-2443

32. Carey PJ, Oberst MT, McCubbin MA, Hughes SH. Appraisal and caregiving burden in family members caring for patients receiving chemotherapy. Oncol Nurs Forum. 1991; 18(8):1341-8. [PubMed: 1762974]

33. Oberst MT, Hughes SH, Chang AS, McCubbin MA. Self-care burden, stress appraisal, and mood among persons receiving radiotherapy. Cancer Nurs. 1991; 14(2):71-8. [PubMed: 2044064]

34. Bakas T, Champion V. Development and psychometric testing of the Bakas Caregiving Outcomes Scale. Nurs Res. 1999; 48(5):250-9. [PubMed: 10494909]

35. Williams LS, Bakas T, Brizendine E, et al. How valid are family proxy assessments of stroke patients' health-related quality of life? Stroke. 2006; 37(8):2081-5. [PubMed: 16809575]

36. SAS Institute, Inc.. SAS/STAT® 9.3 User's Guide. SAS Institute Inc.; Cary, NC: 2011.

37. Hosmer, D.; Lemeshow, S. Applied Logistic Regression. John Wiley \& Sons; New York, NY: 1989.

38. Benjamini Y, Hochberg Y. Controlling the false discovery rate: a practical and powerful approach to multiple testing. J R Stat Soc Ser B. 1995; 57:289-300.

39. Benjamini Y, Yekutieli D. The control of the false discovery rate in multiple testing under dependency. Ann Stat. 2001; 29:1165-1188.

40. Pearson WS, Bhat-Schelbert K, Probst JC. Multiple chronic conditions and the aging of America: Challenge for primary care physicians. J Prim Care Community Health. 2012; 3(1):51-6. [PubMed: 23804855]

41. White CL, Mayo N, Hanley JA, Wood-Dauphinee S. Evolution of the caregiving experience in the initial 2 years following stroke. Res Nurs Health. 2003; 26(3):177-89. [PubMed: 12754726]

42. Teel C, Duncan P, Lai S. Caregiving experiences after stroke. Nurs Res. 2001; 50(1):53-60. [PubMed: 19785245]

43. Rigby H, Gubitz G, Eskes G, et al. Caring for stroke survivors: baseline and 1-year determinants of caregiver burden. Int J Stroke. 2009; 4(3):152-8. [PubMed: 19659814]

44. Tiegs TJ, Heesacker M, Ketterson TU, et al. Coping by stroke caregivers: Sex similarities and differences. Top Stroke Rehabil. 2006; 13(1):52-62. [PubMed: 16581630]

45. Rouillard S, DeWeerdt W, DeWit L, Jelsma JS. Functioning at 6 months post stroke following discharge from inpatient rehabilitation. Afr Med J. 2012; 102(6):545-8. 23.

46. Clark PC, Dunbar SB, Shields CG, Viswanathan B, Aycock DM, Wolf SL. Influence of stroke survivor characteristics and family conflict surrounding recovery on caregivers' mental and physical health. Nurs Res. 2004; 53(6):406-13. [PubMed: 15586137] 


\section{Table 1}

Sample Characteristics $(\mathrm{N}=242)$

\begin{tabular}{lllc}
\hline Variable & Non-Missing & Mean (SD) or Frequency (\%) & Range \\
\hline Caregiver Age (years) & $238(98 \%)$ & $54.2(12.1)$ & $22-87$ \\
Caregiver Chronic Conditions & $242(100 \%)$ & $2.20(1.77)$ & $0-9$ \\
Caregiver Gender & $242(100 \%)$ & & N/A \\
$\quad$ Female & & $191(78.6 \%)$ & \\
$\quad$ Male & $241(99 \%)$ & $52(21.4 \%)$ & N/A \\
Caregiver Race & & $173(71.8 \%)$ & \\
$\quad$ White & & $59(24.5 \%)$ & \\
$\quad$ African-American & & $9(3.7 \%)$ & \\
$\quad$ Other & $242(100 \%)$ & & $0-25$ \\
Caregiver Relationship & & $127(52.3 \%)$ & \\
$\quad$ Non-Spouse & & $116(47.7 \%)$ & 15 - \\
$\quad$ Spouse & $242(100 \%)$ & $5.35(4.85)$ & $19-93$ \\
Caregiver Depressive Symptoms (PHQ9) & $126(52 \%)$ & $1.79(1.49)$ & $25-94$ \\
$\quad$ No Depressive Symptoms (<5) & $116(48 \%)$ & $9.22(4.24)$ & $0-9$ \\
$\quad$ Mild to Severe Depressive Symptoms ( 25$)$ & $242(100 \%)$ & $31.1(11.5)$ & \\
Caregiving Task Difficulty (OCBS) & $242(100 \%)$ & $56.1(10.4)$ & $63.0(14.1)$ \\
Caregiving Life Changes (BCOS) & $228(94 \%)$ & $4.08(1.68)$ & \\
Survivor Age (years) & $242(100 \%)$ & $3.32(1.06)$ & \\
Survivor Chronic Conditions & $242(100 \%)$ & $2.95(1.04)$ & \\
Survivor Mobility (SSQOL) & $242(100 \%)$ & & \\
Survivor Thinking (SSQOL) & & & \\
\hline
\end{tabular}


Table 2

Results Comparing Depressive Symptoms Groups (PHQ9): Testing for Covariates

\begin{tabular}{|c|c|c|c|c|}
\hline Variable & $\begin{array}{r}\text { No Depressive Symptoms } \\
\text { Group Mean }(S D) \text { or Frequency } \\
(\%)\end{array}$ & $\begin{array}{r}\text { Mild to Severe Depressive } \\
\text { Symptoms Group Mean }(S D) \text { or } \\
\text { Frequency }(\%)\end{array}$ & $\begin{array}{r}\text { Test Statistic (DF) T-Test or } \\
\text { Chi Squared }\end{array}$ & $p$ \\
\hline Caregiver Age (years) & $54.8(12.2)$ & $53.3(11.8)$ & $0.95(235)$ & .345 \\
\hline Caregiver Chronic & $1.90(1.59)$ & $2.49(1.89)$ & $-2.62(240)$ & .009 \\
\hline \multicolumn{5}{|l|}{ Conditions } \\
\hline Caregiver Gender & & & $14.5(1)^{l}$ & $<.001$ \\
\hline Female & $87(69 \%)$ & $103(89 \%)$ & & 1 \\
\hline Male & $39(31 \%)$ & $13(11 \%)$ & & \\
\hline Caregiver Race & & & $4.1(2)^{l}$ & $.129^{l}$ \\
\hline White & $88(71 \%)$ & $84(72 \%)$ & & \\
\hline African-American & $34(27 \%)$ & $25(22 \%)$ & & \\
\hline Other & $2(2 \%)$ & $7(6 \%)$ & & \\
\hline Caregiver Relationship & & & $1.28^{1}$ & $.303^{1}$ \\
\hline Non-Spouse & $70(56 \%)$ & $56(48 \%)$ & & \\
\hline Spouse & $56(44 \%)$ & $60(52 \%)$ & & \\
\hline Survivor Age (years) & $62.8(15.1)$ & $63.3(13.0)$ & $-0.28(225)$ & .781 \\
\hline Survivor Chronic & $3.93(1.58)$ & $4.25(1.78)$ & $-1.49(240)$ & .139 \\
\hline \multicolumn{5}{|l|}{ Conditions } \\
\hline Survivor Mobility & $3.53(1.05)$ & $3.08(1.04)$ & 3.35 (239) & $<.001$ \\
\hline Survivor Thinking & $3.22(1.06)$ & $2.66(0.94)$ & $4.29(240)$ & $<.001$ \\
\hline
\end{tabular}


Table 3

General Linear Model Results for Task Difficulty (OCBS) and Life Changes (BCOS) based on Depressive Symptoms Groups (PHQ9)

\begin{tabular}{lccc}
\hline OCBS Total $\left(\mathbf{R}^{2}=. \mathbf{2 3 6}\right)$ & Mean Square & $\mathbf{F}(\mathbf{D F})$ & $\mathbf{p}$ \\
Overall Model & 1501.4 & $14.49(5,235)$ & $<.001$ \\
PHQ9 Group & 1720.8 & $16.61(1,235)$ & $<.001$ \\
Caregiver Gender & 193.6 & $1.87(1,235)$ & .173 \\
Caregiver Chronic Conditions & 133.4 & $1.29(1,235)$ & .258 \\
Survivor Mobility & 1664.8 & $16.07(1,235)$ & $<.001$ \\
Survivor Thinking & 643.3 & $6.21(1,235)$ & .013 \\
Error & 103.6 & & \\
BCOS Total $\left(\mathbf{R}^{2}=.210\right)$ & Mean Square & $\mathbf{F}(\mathbf{D F})$ & $\mathbf{p}$ \\
Overall Model & 1086.8 & $12.40(5,234)$ & $<.001$ \\
PHQ9 Group & 2216.1 & $25.29(1,234)$ & $<.001$ \\
Caregiver Gender & 19.5 & $0.22(1,234)$ & .637 \\
Caregiver Chronic Conditions & 309.5 & $3.53(1,234)$ & .061 \\
Survivor Mobility & 191.0 & $2.18(1,234)$ & .141 \\
Survivor Thinking & 655.9 & $7.49(1,234)$ & .007 \\
Error & 87.6 & & \\
\hline
\end{tabular}




\section{Table 4}

Differences in Individual Task Difficulty (OCBS) and Life Changes (BCOS) Items based on Depressive Symptoms Groups (PHQ9) (N = 242)

\begin{tabular}{|c|c|c|}
\hline$\underline{\text { OCBS Item (\#) }}$ & Odds Ratio (95\% CI) & $\underline{p}$ \\
\hline Arranging care while away (12) & $3.456(1.827-6.706)$ & $<.001^{*}$ \\
\hline Providing personal care (2) & $2.852(1.450-5.777)$ & .003 \\
\hline Giving emotional support (4) & $2.438(1.282-4.696)$ & .007 \\
\hline Watching and monitoring the patient (5) & $2.472(1.268-4.937)$ & .009 \\
\hline Talking with health care providers (15) & $2.175(1.162-4.150)$ & .016 \\
\hline Assisting with walking (3) & $2.313(1.125-4.885)$ & .024 \\
\hline Coordinating care (14) & $2.063(1.070-4.035)$ & .032 \\
\hline Providing transportation (6) & $1.954(1.023-3.771)$ & .044 \\
\hline Tasks outside home (9) & $1.858(.970-3.598)$ & .063 \\
\hline Medical or nursing treatments (1) & $1.845(.969-3.571)$ & .065 \\
\hline Planning activities (10) & $1.767(.954-3.307)$ & .072 \\
\hline Managing behavior problems (11) & $1.789(.935-3.445)$ & .080 \\
\hline Household tasks (8) & $1.733(.909-3.321)$ & .095 \\
\hline Managing finances (7) & $1.481(0775-2.844)$ & .235 \\
\hline Communication problems (13) & $1.369(.706-2.666)$ & .352 \\
\hline BCOS Item (\#) & Odds Ratio (95\% CI) & p \\
\hline Self-esteem (1) & $7.217(2.771-22.82)$ & $<.001^{*}$ \\
\hline Ability to cope with stress (4) & $4.035(2.062-8.196)$ & $<.001^{*}$ \\
\hline Physical health (2) & $3.790(1.913-7.874)$ & $<.001^{*}$ \\
\hline Emotional well-being (8) & $3.327(1.729-6.402)$ & $<.001^{*}$ \\
\hline Financial well-being (12) & $3.092(1.623-6.012)$ & $<.001 *$ \\
\hline Level of energy (7) & $2.998(1.585-5.669)$ & $<.001^{*}$ \\
\hline General health (15) & $3.371(1.640-7.287)$ & $.001^{*}$ \\
\hline Roles in life (9) & $2.883(1.481-5.774)$ & $.002 *$ \\
\hline Future outlook (6) & $2.676(1.369-5.378)$ & $.005^{*}$ \\
\hline Physical functioning (14) & $2.536(1.272-5.242)$ & $.010^{*}$ \\
\hline Relationship with friends (5) & $2.082(1.099-4.020)$ & .026 \\
\hline Relationship with stroke survivor (13) & $2.367(1.121-5.222)$ & .027 \\
\hline Time for social activities (10) & $2.076(1.075-4.062)$ & .031 \\
\hline Time for family activities (3) & $1.962(1.025-3.795)$ & .043 \\
\hline Relationship with family (11) & $1.950(0.970-4.031)$ & .064 \\
\hline
\end{tabular}

Odds ratios are based on the following:

1.Selecting Moderate, Very, or Extremely Difficult (OCBS items rated as 3, 4, or 5) and Changed for the Worse (BCOS items rated as 1, 2, or 3 on a scale from 1 to 7 ).

2.No depressive symptoms (PHQ $9<5$ ) group as referent group.

3.Profile-likelihood after controlling for caregiver gender, caregiver chronic conditions, survivor mobility, and survivor thinking covariates.

* Meets Benjamini-Hochberg false discovery rate criterion 